\title{
Combination therapy with dutasteride and tamsulosin for the treatment of symptomatic enlarged prostate
}

This article was published in the following Dove Press journal:

Clinical Interventions in Aging

28 May 2009

Number of times this article has been viewed

\author{
J Miller \\ TH Tarter \\ Southern Illinois University School \\ of Medicine, Department \\ of Surgery, Springfield, IL, USA
}

Correspondence:Thomas H Tarter Division of Urology, Southern Illinois University School of Medicine, PO Box 19665,

Springfield, IL 62794-9665, US.

Tel +I 2175457390

Fax +I 2175457305

Email ttarter@siumed.edu

\begin{abstract}
Benign prostatic hyperplasia (BPH) is a frequent cause of lower urinary symptoms, with a prevalence of $50 \%$ by the sixth decade of life. Hyperplasia of stromal and epithelial prostatic elements that surround the urethra cause lower urinary tract symptoms (LUTS), urinary tract infection and acute urinary retention. Medical treatments of symptomatic BPH include; 1) the $5 \alpha$-reductase inhibitors, 2) the $\alpha 1$-adrenergic antagonists, and 3) the combination of a $5 \alpha$-reductase inhibitor and a $\alpha 1$-adrenergic antagonist. Selective $\alpha 1$-adrenergic antagonists relax the smooth muscle of the prostate and bladder neck without affecting the detrussor muscle of the bladder wall, thus decreasing the resistance to urine flow without compromising bladder contractility. Clinical trials have shown that $\alpha 1$-adrenergic antagonists decrease LUTS and increase urinary flow rates in men with symptomatic $\mathrm{BPH}$, but do not reduce the long-term risk of urinary retention or need for surgical intervention. Inhibitors of $5 \alpha$-reductase decrease production of dihydrotestosterone within the prostate resulting in decreased prostate volumes, increased peak urinary flow rates, improvement of symptoms, and decreased risk of acute urinary retention and need for surgical intervention. Interim results of the ongoing Combination of Avodart and Tamsulosin (CombAt) study have shown combination therapy with the $5 \alpha$-reductase inhibitor dutasteride and the $\alpha 1$-adrenergic antagonist tamsulosin offer significant improvements from baseline compared with either drug alone.
\end{abstract}

Keywords: prostatic hyperplasia, $5 \alpha$-reductase, dutasteride, tamsulosin

\section{Introduction}

Benign prostatic hyperplasia $(\mathrm{BPH})$ refers to stromal and glandular epithelial hyperplasia that occurs in the zone of the prostate that surrounds the urethra. In the absence of histopathology, the clinical term benign prostatic enlargement (BPE) is used to describe the presumed etiology of associated lower urinary tract symptoms (LUTS), including urinary frequency and urgency, a sensation of incomplete bladder emptying, a weak and interrupted urinary stream, straining to initiate urination, and nocturia. The prevalence of LUTS due to BPH/BPE increases with increasing age, and moderate to severe symptoms occur in up to $40 \%$ of men after age 60 . Symptoms are evaluated with validated instruments such as the American Urologic Association (AUA) Symptom Index and the International Prostate Symptom Score (IPSS). Each of seven symptoms (frequency, urgency, weak stream, intermittency, incomplete emptying, straining to urinate, and nocturia) are scored by the patient on a $0-5$ scale based on their frequency. A score of less than 7 indicates mild symptoms; a score of 8-19 indicates moderate symptoms, and a score of greater than 19 indicates severe symptoms. In addition to symptoms that may have a negative impact on the quality of life, $\mathrm{BPH} / \mathrm{BPE}$ can result in acute urinary retention, 
recurrent urinary tract infections (UTI), bladder stones, urinary incontinence, gross hematuria and renal failure.

The natural history of BPH/BPE is unpredictable in individual men. In a study of men who were followed expectantly for 5 years without treatment, $31 \%$ reported symptomatic improvement whereas $16 \%$ reported symptomatic worsening. ${ }^{1}$ Men with symptomatic BPH/BPE have a $23 \%$ lifetime risk of developing acute urinary retention if left untreated. ${ }^{2}$ A man over age 60 years with obstructive symptoms has a $39 \%$ probability of undergoing surgery related to the prostate within 20 years. ${ }^{3}$

The American Urological Association and the European Association of Urology have published recommendations for the evaluation of men with LUTS, and the treatment of men with symptomatic BPH/BPE. Medical therapies recommended by these two organizations include the $\alpha 1$-adrenergic antagonists terazosin, doxazosin, tamsulosin, and alfuzosin, and the 5 $\alpha$-reductase inhibitors finasteride and dutasteride. ${ }^{4}$

Selective $\alpha 1$-adrenergic antagonists relax the smooth muscle of the prostate and bladder neck without affecting the detrusor muscle of the bladder wall, thus decreasing the resistance to urine flow without compromising bladder contractility. Randomized, placebo-controlled clinical trials have shown that $\alpha 1$-adrenergic antagonists decrease LUTS and increase urinary flow rates in men with symptomatic BPH/BPE. However, a positive placebo effect was also demonstrated for both symptom score and peak urinary flow rates in these trials. Common side effects include dizziness, headache, asthenia and postural hypotension, which occur in $5 \%$ to $9 \%$ of patients. ${ }^{5}$ Tamsulosin is the most uroselective $\alpha 1$-adrenergic antagonist approved for use in the treatment of symptomatic BPH/BPE. Clinical trials have shown postural hypotension was observed less frequently with tamsulosin than with either terazosin or doxazosin. ${ }^{6}$

Dihydrotestosterone (DHT) is the product of the conversion of testosterone by the enzyme $5 \alpha$-reductase, and is produced in the tissues of the liver, skin and organs that originate from the mesonephric duct, such as the prostate. Within the prostate, locally produced DHT acts in a paracrine fashion to stimulate growth. Inhibitors of $5 \alpha$-reductase decrease production of DHT within the prostate resulting in decreased prostate volume, increased peak urinary flow rates, and improvement in symptom scores. Studies have also shown that $5 \alpha$-reductase inhibitors reduce serum levels of prostate specific antigen and reduce the overall risk of prostate cancer. ${ }^{7}$ Side effects of $5 \alpha$-reductase inhibitors include erectile dysfunction, decreased libido, ejaculatory dysfunction and gynecomastia, which occur in less than $5 \%$ of patients. ${ }^{8}$ Dutasteride inhibits both type 1 and type $25 \alpha$-reductase isoenzymes, whereas finasteride inhibits only type 2 .

Combination therapy with the $\alpha 1$-adrenergic antagonist doxazosin and the $5 \alpha$-reductase inhibitor finasteride has been shown in previous trials to significantly reduce the overall risk of clinical progression of BPH/BPE compared to the use of either drug alone. Herein we detail the use of the $5 \alpha$-reductase inhibitor dutasteride and the $\alpha 1$-adrenergic antagonist tamsulosin, and review the role of combination therapy in the medical treatment of LUTS caused by prostatic enlargement.

\section{Pharmacology, mode of action, and pharmacokinetics of dutasteride}

Dutasteride is in a drug class known as $17 \beta$-substituted 4-aza-steroids with the chemical name $(5 \alpha, 17 \beta)-\mathrm{N}\{2,5$, bis(trifluoromethyl) phenyl $\}$-3-oxo-4-azaandrost-1-ene17-carboxamide. The structural formula is shown in Figure 1. It is a competitive inhibitor of type 1 and type $25 \alpha$-reductase isoenzymes. It forms a stable complex with a slow rate of dissociation, and does not bind the androgen receptor. The bioavailability of dutasteride is approximately $60 \%$ and peak serum concentration is achieved after 2 to 3 hours. The time to steady state is dose dependent, and at a dose of $0.5 \mathrm{mg} /$ day is approximately 3 months. More than $99.5 \%$ of circulating dutasteride is bound to plasma proteins, and has a volume of distribution of 300 to $500 \mathrm{~L}$. Clearance is linear at $0.58 \mathrm{~L} /$ hour, resulting in a half-life of up to 5 weeks. Pharmacokinetic data are summarized in Table 1. Dutasteride is detectable (greater than $0.1 \mathrm{ng} / \mathrm{mL}$ ) in the serum 4 to 6 months after discontinuation of treatment. The drug is extensively metabolized by cytochrome-P3A4 in the liver and is excreted mainly in the feces. Only trace amounts

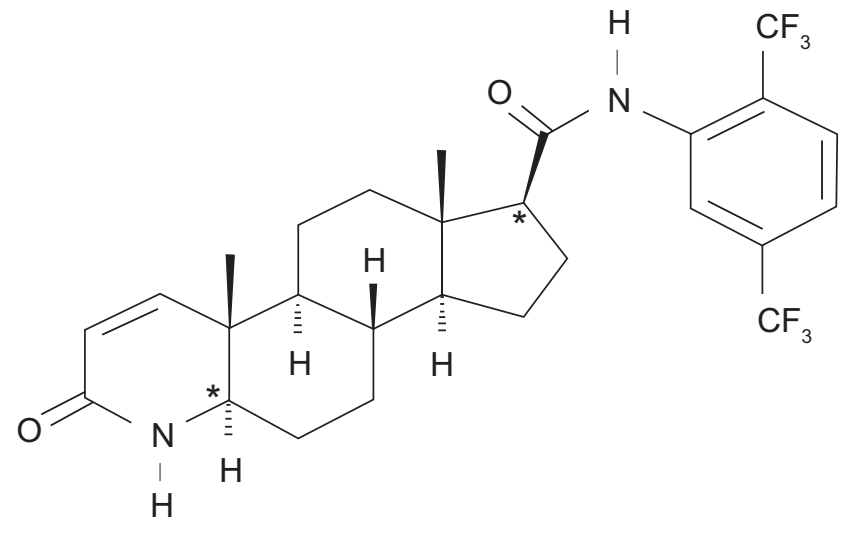

Figure I Dutasteride chemical structure. 
Table I Pharmacokinetics of dutasteride

\begin{tabular}{ll}
\hline Bioavailability & $60 \%$ \\
\hline Steady state & 3 months \\
Peak serum concentration & $2-3$ hours \\
Volume of distribution & $511 \mathrm{~L}$ \\
Elimination half-life & 5 weeks \\
\hline
\end{tabular}

are excreted in the urine. Following dosing to steady state, 5 major metabolites are detectable in the serum. The major metabolite, 6 $\beta$-hydroxydutasteride, has pharmacological activity comparable to dutasteride. ${ }^{9}$

Caution should be used when administering the drug in patients with hepatic impairment, and in patients who take drugs that inhibit cytochrome-P3A4 (verapamil, diltiazem, and ritonavir) because the pharmacokinetics and risk of side effects may be altered. No other significant drug interactions have been identified.

In three pooled 2-year studies, the most frequent side effects were impotence, decreased libido, gynecomastia, and ejaculatory dysfunction. The incidence of side effects compared to placebo was increased from $4 \%$ to $7.3 \%$ for impotence, $2.1 \%$ to $4.2 \%$ for decreased libido, $0.7 \%$ to $2.3 \%$ for gynecomastia, and $0.8 \%$ to $2.2 \%$ for ejaculatory dysfunction. ${ }^{10}$

No dose adjustment is necessary in the elderly. Of 2617 men treated with dutasteride in three pivotal studies, $60 \%$ were age 65 and over and 15\% were age 75 and over. In these studies no overall difference in safety or efficacy was observed between older and younger patients. No change in bone mineral density as measured on dual energy x-ray absorptiometry was observed compared with placebo. Plasma lipid profiles were unaffected. ${ }^{11}$

Dutasteride is not indicated for use in women and the pharmokinetics have not been investigated in patients younger than 18 years. Due to potential adverse effects on normal development of the male reproductive tract, use of dutasteride should be avoided in men less than 18 years of age, and is absolutely contraindicated in women of reproductive age. ${ }^{11}$

\section{Efficacy and safety of dutasteride}

The efficacy and safety of dutasteride was studied in three parallel, randomized, placebo-controlled trials over a period of 2 years. A total of 4325 men age 50 years or older with moderate to severe symptomatic BPH/BPE (AUA symptom score of 12 or more and peak urinary flow rate of $15 \mathrm{~mL} / \mathrm{sec}$ or less) and prostate volume greater than $30 \mathrm{~cm}^{3}$ were randomized to receive either dutasteride $0.5 \mathrm{mg} /$ day or placebo. Endpoints included changes in prostate volume, peak urinary flow rate, risk of surgical intervention, safety and tolerability, and measurements of serum PSA, testosterone, and DHT. ${ }^{10}$

The mean improvement in symptom score in the placebo groups was $2.3( \pm 6.8)$ to $14.7( \pm 7.2)$ from a baseline of 17.1 $( \pm 6.1, \mathrm{p}<0.001)$. In the dutasteride groups, a 4.5-point $( \pm 6.6)$ improvement to a mean of $12.2( \pm 6.6)$ was significant compared to baseline of $17.0( \pm 6.0, p<0.001)$ and compared with placebo groups $(\mathrm{p}<0.001)$. Peak urinary flow rates in the placebo groups increased by $0.6 \mathrm{~mL} / \mathrm{sec}( \pm 4.7)$ to $11.2 \mathrm{~mL} / \mathrm{sec}( \pm 4.8)$, which was significant compared with baseline of $10.4 \mathrm{~mL} / \mathrm{sec}( \pm 3.6, \mathrm{p}<0.001)$. Peak urinary flow rates in the dutasteride groups increased by a mean of $2.2 \mathrm{~mL} / \mathrm{sec}( \pm 5.2)$ to $12.5( \pm 5.6)$, which was significant compared to baseline of $10.1 \mathrm{~mL} / \mathrm{sec}( \pm 3.5, \mathrm{p}<0.001)$, and to the placebo groups ( $\mathrm{p}<0.001$ ). After 24 months of therapy, the mean total prostate volume increased by $12.4 \%$ in the placebo groups and decreased by $20.4 \%$ in the dutasteride groups $(\mathrm{p}<0.001)$. The relative risk of acute urinary retention in the dutasteride groups compared with the placebo groups was 0.43 , or a risk reduction of $57 \%$ $(p<0.001)$. The relative risk of surgical intervention in the dutasteride groups compared to the placebo groups was 0.52 , or a risk reduction of $48 \%(\mathrm{p}<0.001)$. Significant changes in serum DHT $(16( \pm 150)$ vs $-389( \pm 228) \mathrm{pg} / \mathrm{mL})$ and PSA $(0.5( \pm 2.1) \mathrm{vs}-2.2( \pm 2.0) \mathrm{ng} / \mathrm{mL})$, as well as a significantly larger increase in serum testosterone $(36( \pm 1226)$ vs 749 $( \pm 1475) \mathrm{pg} / \mathrm{mL})$ were noted in the dutasteride groups compared to the placebo groups ( $p<0.001$ for each comparison). ${ }^{10}$

Adverse events attributed to the study drug were reported in $14 \%$ of men in the placebo groups and in $19 \%$ of men in the dutasteride groups. A higher proportion of men in the dutasteride groups than in the placebo groups reported impotence, reduced libido, ejaculatory dysfunction, and gynecomastia. ${ }^{10}$ Results are summarized in Table 2.

Data from several open-label extensions of the above three parallel, randomized placebo-controlled trials have

Table 2 Sexual adverse events from dutasteride efficacy and safety study

\begin{tabular}{lll}
\hline Adverse event & Placebo & Dutasteride \\
\hline Impotence & $4.00 \%$ & $7.30 \%$ \\
Decreased libido & $2.10 \%$ & $4.20 \%$ \\
Gynecomastia & $0.70 \%$ & $2.30 \%$ \\
Ejaculation disorder & $0.80 \%$ & $2.20 \%$ \\
\hline
\end{tabular}


recently been reported. These studies show continued tolerability and efficacy after 48 months of treatment. In these trials less than $1 \%$ of the men withdrew from the open-label extension because of sexual dysfunction. Approximately 1\% of men withdrew because of gynecomastia. Patients who received dutasteride during both study phases showed greater improvement in symptom scores and urinary peak flow rates than those initially receiving placebo..$^{5,12,13}$

\section{Effect of dutasteride on quality of life}

Data regarding the impact on quality of life of the 4325 men in the three randomized, double-blind, placebo controlled, 2-year studies detailed above have also been reported. At 2 years, dutasteride, but not placebo, resulted in clinically and statistically significant improvements from baseline in the BPH Impact Index (BII) score after 6 months of treatment. This net improvement from baseline increased from 6 months to 2 years. ${ }^{14}$

Additional data were recently reported from a separate prospective, multi-center, open-label study evaluating the improvement in symptoms and quality of life in patients with symptomatic BPH/BPE treated with dutasteride. Patients received dutasteride $0.5 \mathrm{mg} /$ day for 24 weeks. The primary endpoint was the proportion of patients who achieved at least a 3-point decrease from baseline in IPSS. The secondary endpoints were changes in quality of life (IPSS item 8) and patient discomfort and satisfaction, assessed using the visual analogue scale (VAS).

A total of 366 patients from 72 centers were included in the study. After 24 weeks of treatment with dutasteride, $72.5 \%$ of patients achieved at least a 3-point reduction in IPSS. The mean IPSS score decreased from $15.3( \pm 6.4)$ at baseline to $9.1( \pm 5.6, p<0.001)$ at 24 weeks. Patients with more severe symptoms had a higher probability of improvement in the IPSS. There were statistically significant decreases in all individual IPSS items, with greater improvement seen in items related to irritative and obstructive voiding symptoms than in those items related to storage symptoms.

Patient quality of life improved as assessed by IPSS item 8 with a mean improvement of $38.7 \%$ from baseline $(\mathrm{p}<0.001)$. Patient discomfort and satisfaction also significantly improved. The mean VAS for discomfort decreased from 48.9 at baseline to 28.6 ( $p<0.001)$ at 24 weeks. Patient VAS scores for satisfaction increased from a baseline at both 12 and 24 weeks ( $<<0.001)$.

Overall, 77 (19\%) patients had at least one adverse event related to dutasteride during the study period, including erectile dysfunction (7\%), decreased libido (4\%), and gynecomastia $(2 \%){ }^{15}$

\section{Dutasteride in prostate cancer prevention and treatment}

An in depth discussion of the role of $5 \alpha$-reductase inhibitors in the prevention and treatment of prostate cancer is beyond the scope of this review; however the clinical implications of long-term use of finasteride or dutasteride in the treatment of BPH must be considered in light of new information from recent trials.

In the Prostate Cancer Prevention Trial (PCPT) 18882 men age 55 years or older with a PSA of $3.0 \mathrm{ng} / \mathrm{mL}$ or less were randomized to receive finasteride $5 \mathrm{mg}$ or placebo daily for 7 years. Prostate biopsies were performed at the end of the 7-year study period, or if the annual PSA level (adjusted for the effect of finasteride) was greater than $4 \mathrm{ng} / \mathrm{mL}$, or if the digital rectal exam (DRE) was suspicious for prostate cancer. The primary endpoint measure was the diagnosis of prostate cancer. The study was terminated 15 months early on the recommendations of the data and safety monitoring committee because the study objective had been met. Prostate cancer was detected in $18.4 \%$ of men in the finasteride group and in $24.4 \%$ of men in the placebo group, a relative risk reduction of $24.8 \%(\mathrm{p}<0.001)$. However, of the men who developed prostate cancer, high-grade prostate cancer was reported in a higher proportion of men in the finasteride group than in the placebo group $(37 \%$ vs $22 \%$, p $<0.001){ }^{7}$ Androgen deprivation therapy, including inhibition of DHT by finasteride, results in specific histopathologic changes in prostatic adenocarinoma, which can result in a "grading bias." In the PCPT, most of the high-grade cancer were reported in the earlier phase of the study, a finding that is at odds with the development of more aggressive cancers with prolonged finasteride treatment. ${ }^{16}$ At the present time FDA-labeled indications in the United States for finasteride and dutasteride do not include prostate cancer prevention.

An ongoing study, the Reduction by Dutasteride of Prostate Cancer Events (REDUCE) Trial will use dutasteride in a group of men identified at increased risk of developing prostate cancer to determine if this dual $5 \alpha$-reductase inhibitor will be an effective chemoprevention agent. Eligible men must be between ages 50 and 75 and have a PSA between $2.5 \mathrm{ng} / \mathrm{mL}$ and $10 \mathrm{ng} / \mathrm{ml}$ (age 50-60 years) or between $3.0 \mathrm{ng} / \mathrm{mL}$ and $10 \mathrm{ng} / \mathrm{mL}$ (age $>60$ years), and a free PSA of $25 \%$ or lower at baseline, and a negative prostate biopsy. The men are randomized to placebo or dutasteride $0.5 \mathrm{mg}$ daily. Prostate biopsies will be performed at 2 years and at 4 years to evaluate for prostate cancer. Additionally, free and 
total PSA will be measured every 6 months, with adjustment of the PSA of patients randomized to the dutasteride arm. The primary endpoint is biopsy-proven prostate cancer after 2 and 4 years of treatment and the study also aims to address the issue of high-grade tumors prospectively. The results of the REDUCE trial will have to be employed with the caution, as all men included in the already had a negative prostate biopsy, which is not the case in clinical practice.

As discussed above, dutasteride inhibits both type 1 and type $25 \alpha$-reductase isoenzymes whereas finasteride inhibits only type 2 . Type $25 \alpha$-reductase is the predominate isoenzyme in normal and hyperplastic prostate tissue. Recent studies have shown that immunohistochemical staining of type 1 is enhanced in prostate cancer tissues compared to BPH epithelium. This suggests that a dual inhibitor of $5 \alpha$-reductase isoenzymes, such as dutasteride, may be a reasonable agent to evaluate for prevention of prostate cancer. ${ }^{17}$

As information from ongoing trials is compiled, physicians who recommend $5 \alpha$-reductase inhibitors for the treatment of patients with symptomatic BPH/BPE should discuss the effect of therapy on 1) reduction in the risk of developing prostate cancer, and 2) the histopathology of prostate biopsies and the potential risk of developing high-grade prostate cancer. Prescribing physicians must also ensure that proper drug-effect corrections are made to serum PSA values and that vigilance is maintained in the form of prostate biopsy, when indicated.

In addition to its potential role in the prevention of prostate cancer, dutasteride has been evaluated as a component of neoadjuvant androgen deprivation therapy before brachytherapy for prostate cancer to reduce prostate volume and facilitate seed implantation. In a recently reported study, 31 patients opted for cytoreductive therapy with bicalutamide $50 \mathrm{mg}$ and dutasteride $0.5 \mathrm{mg}$ daily. All patients underwent transrectal ultrasound volumetric study of the prostate gland, with ellipsoid volume determination of the prostate gland and transition zone, before initiation of the neoadjuvant therapy and at 3 months after initiation. After the 3-month course of combination therapy, the average prostate volume decreased by $33.6 \%$ using the volumetric determination, and decreased by $34.6 \%$ using the ellipsoid volume determination. The average transition zone volume decreased from $20.8 \mathrm{~cm}^{3}$ to $12.4 \mathrm{~cm}^{3}$, a reduction of $39.8 \% .^{18}$ Although these data are not the product of a randomized, placebo-controlled trial, the results are consistent with previous studies of androgen deprivation therapy, and dutasteride may be a reasonable alternative to the use of luteinizing hormone-releasing hormone (LHRH) agonists, which have been associated with more significant side effects.

\section{Pharmacology, mode of action, and pharmacokinetics of tamsulosin}

Tamsulosin hydrochloride is a competitive antagonist of $\alpha_{1 \mathrm{~A}}$ adrenoreceptors with the chemical name (-)-(R)-5[2-[[2-(o-Ethoxyphenoxy) ethyl]amino]propyl]-2-methoxybenzenesulfonamide, monohydrochloride. The structural formula is shown in Figure 2. There are three identified $\alpha_{1}$ adrenoreceptor subtypes found in human organs and tissue: $\alpha_{1 \mathrm{~A}}, \alpha_{1 \mathrm{~B}}$, and $\alpha_{1 \mathrm{D}}$. Approximately $70 \%$ of the $\alpha_{1}$ adrenoreceptors in the human prostate are of the $\alpha_{1 \mathrm{~A}}$ subtype. Under fasting conditions absorption of the orally administered recommended dosage of tamsulosin is greater than $90 \%$. The time to maximum concentration is 4 to 5 hours under fasting condition and 6 to 7 hours when tamsulosin is administered with food. Administration of tamsulosin under fasting conditions results in a $40 \%$ to $70 \%$ increase in peak concentrations compared to the same dosage administered with food. The time to steady state is approximately 5 days. More than $94 \%$ of tamsulosin is bound to circulating plasma proteins and the drug has a volume of distribution of $16 \mathrm{~L}$. Tamsulosin undergoes restrictive clearance with a systemic clearance of $2.88 \mathrm{~L} /$ hour and a half-life of 9 to 15 hours. Pharmacokinetic data are summarized in Table 3.

Tamsulosin is extensively metabolized by cytochromeP3A4 and-P2D6 enzymes in the liver and is primarily (76\%) excreted in the urine with a smaller fraction (21\%) excreted in the feces. The metabolites of tamsulosin are extensively conjugated to glucuronide or sulfate prior to renal excretion. The pharmacokinetics of metabolites have not been established.

Dosage adjustment of tamsulosin is not necessary in patients with mild-to-severe renal impairment $\left(\mathrm{CL}_{\mathrm{cr}} \geq 10\right.$ $\mathrm{mL} / \mathrm{min} / 1.73 \mathrm{~m}^{2}$ ) or mild-to-moderate hepatic dysfunction (Child-Pugh's classification A and B). Tamsulosin has not been studied in patients with end-stage renal disease or severe hepatic dysfunction. Clearance of tamsulosin decreases with age-dependent decreases in plasma binding resulting in a $40 \%$ increased exposure in patients aged 55 to 75 years compared with those aged 20 to 32 years. Tamsulosin should be administered with caution in combination with cimetidine due to

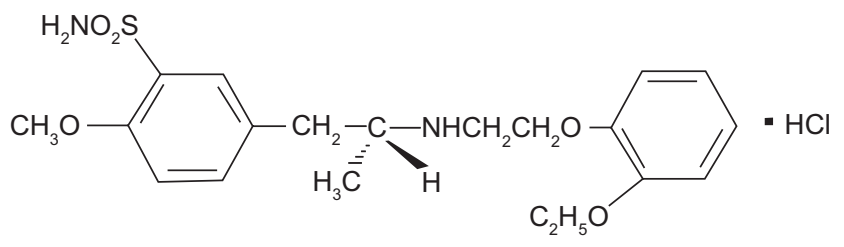

Figure 2 Tamulosin chemical structure. 
Table 3 Pharmacokinetics of tamsulosin

\begin{tabular}{ll}
\hline Bioavailability & $\mathbf{9 0 \%}$ \\
\hline Steady state & 5 days \\
Peak serum concentration & $4-6$ hours \\
Volume of distribution & $16 \mathrm{~L}$ \\
Elimination half-life & 15 hours \\
\hline
\end{tabular}

significant $(26 \%)$ decrease in the clearance of tamsulosin. Dosage adjustments are not required for concomminant administration of tamsulosin and nifedipine, atenolol, enalapril, digoxin, theophylline, or furosemide. Studies of interactions in patients receiving warfarin are inconclusive; therefore tamsulosin should be administered with caution in these patients. Additionally, interactions with moderate to strong inhibitors of CYP3A4 and CYP2D6 such as fluoxetine and ketoconazole, respectively, have not been studied and caution should be exercised.

In six short-term placebo-controlled clinical trials the most commonly reported adverse reactions to tamsulosin were headache, dizziness, rhinitis and abnormal ejaculation. In two of the studies symptomatic postural hypotension occurred in $0.2 \%$ and $0.4 \%$ of patients receiving $0.4 \mathrm{mg}$ and $0.8 \mathrm{mg}$ of tamsulosin daily compared with no patients in the placebo group.

Intraoperative floppy iris syndrome (IFIS) has been observed in some patients undergoing cataract surgery while being treated with $\alpha 1$-blockers, including tamsulosin. Most cases of IFIS occurred in patients currently taking $\alpha 1$-blockers or who had recently discontinued the use $\alpha 1$-blockers, but has been reported in patients who discontinued use of $\alpha 1$-blockers as long as 9 months prior to cataract surgery. Treating ophthalmologists should be prepared for possible modifications to surgical techniques in patients treated with $\alpha 1$-blockers, including tamsulosin. The benefit of discontinuation of $\alpha 1$-blockers prior to cataract surgery has not been established.

\section{Efficacy and safety of tamsulosin}

The efficacy and safety of tamsulosin was studied in a double-blind, placebo-controlled, randomized, multicenter US trial of $0.4 \mathrm{mg}$ and $0.8 \mathrm{mg}$ of tamsulosin daily. A total of 756 men aged 45 years or older with signs and symptoms of BPH/BPE (AUA symptom score $\geq 13$ or peak urinary flow rate $4-15 \mathrm{~mL} / \mathrm{s}$ ) were randomized to tamsulosin $0.4 \mathrm{mg}$ daily, tamsulosin $0.8 \mathrm{mg}$ daily, or placebo for a total of 13 weeks. Primary end point measures were changes in AUA symptom score, percentage of patients with at least $25 \%$ improvement in AUA symptom score, change in peak urinary flow rate, and percentage of patients with at least 30\% improvement in peak urinary flow rate. Safety was assessed via adverse event profiles, orthostatic vital sign measurements, laboratory testing, EKG findings and physical examination. ${ }^{6}$

The mean improvement in the AUA symptom score in the placebo group was $-5.5( \pm 6.3)$ from a mean baseline of $19.6( \pm 4.9)$ compared with $-8.3( \pm 6.3, \mathrm{p}<0.001)$ in the $0.4 \mathrm{mg} /$ day tamsulosin group and $-9.6( \pm 6.2, \mathrm{p}<0.001)$ in the $0.8 \mathrm{mg} /$ day tamsulosin group ( $\mathrm{p}=0.020 \mathrm{vs} 0.4 \mathrm{mg} /$ day). Peak urinary flow rates increased a mean of $0.53 \mathrm{~mL} / \mathrm{s}( \pm 3.3)$ in the placebo group and $1.75 \mathrm{~mL} / \mathrm{s}( \pm 3.5)$ and $1.78 \mathrm{~mL} / \mathrm{s}$ $( \pm 3.3)$ in the $0.4 \mathrm{mg} /$ day and $0.8 \mathrm{mg} /$ day groups, respectively $(\mathrm{p}<0.001)$. Among patients in the $0.8 \mathrm{mg} /$ day group $74 \%$ achieved a $\geq 25 \%$ improvement in AUA symptom score compared with $70 \%$ in the $0.4 \mathrm{mg} /$ day group and $51 \%$ in the placebo group ( $p<0.001 \mathrm{vs} 0.4 \mathrm{mg} /$ day and $0.8 \mathrm{mg} /$ day). More than $30 \%$ of patients in both tamsulosin groups had $\geq 30 \%$ improvement in peak urinary flow rates compared to $21 \%$ of patients in the placebo group. ${ }^{6}$

There were no clinically significant differences in hematologic tests, EKG results or coagulation parameters in patients receiving tamsulosin compared to patients receiving placebo. Three patients (one in the placebo group, two in the tamsulosin groups) were withdrawn from the study due to elevation of liver function test values. No clinically significant differences in orthostatic changes to systolic or diastolic blood pressure or pulse were found between treatment and placebo groups. ${ }^{6}$

The long-term efficacy and safety of tamsulosin were reported in a four year extension, multicenter, open-label, phase IIIB clinical study which included patients treated up to 6 years with tamsulosin. A total of 609 men aged 45 to 75 years were included, with 109 patients completing 6 years of treatment and 419 completing 5 years of treatment. There was continued and sustained improvement in baseline parameters including statistically significant yearly improvements in AUA symptom score and significant increases in peak urinary flow rates. Compared to shorter-term studies the incidence of most adverse events was not increased. ${ }^{19}$

\section{Combination therapy with dutasteride and tamsulosin}

In 2003 data from the Medical Therapy of Prostate Symptoms (MTOPS) Trial, a study of 3047 men with moderate to severe symptomatic BPH/BPE, showed the risk of clinical progression of BPH/BPE was reduced by $39 \%$ in patients treated with doxazosin alone, by $34 \%$ in patients treated with finasteride alone, and by $66 \%$ in patients treated with 
combination therapy. Similarly, improvements in AUA symptom scores were significantly greater in the combination therapy group than either the doxazosin group or the finasteride group. Peak urinary flow rates were improved and the risk of surgical intervention was reduced in all treatment groups compared with placebo. ${ }^{20}$

More recently, Roehrborn et al published the preplanned two-year results of the Combination of Avodart and Tamsulo$\sin$ (CombAt) study. Like the MTOPS trial, the CombAT study compares the effect of dutasteride alone and tamsulosin alone to the outcomes of combination therapy, but with some important differences. The CombAT study includes only men at increased risk for progression of BPH due to a prostate volume $\geq 30 \mathrm{~mL}$ or a PSA $\geq 1.5 \mathrm{ng} / \mathrm{mL}$; these patients represent a subset population identified by the MTOPS Trial. Additionally, the MTOPS

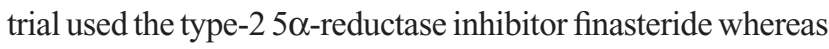
the CombAT study assesses the results of treatment using the dual $5 \alpha$-reductase inhibitor dutasteride. ${ }^{21}$

The CombAT study is an ongoing 4-year randomized, double-blind parallel group study involving 446 investigators in 35 countries. The aim of the study is to investigate the longterm clinical outcomes of patients with BPH/BPE treated with dutasteride alone, tamsulosin alone, and combination therapy. A total of 4838 subjects have been enrolled. The primary endpoint at the end of the initial 2-year study period was the change in IPSS from baseline. Secondary endpoints included IPSS responders ( $\geq 25 \%, \geq 2$-point, and $\geq 3$-point improvement), peak urinary flow rate, peak urinary flow rate responders ( $\geq 30 \%$ and $\geq 3 \mathrm{~mL} / \mathrm{s}$ improvement), total prostate volume, and prostate transition zone volume. At 2 years the mean decrease from IPSS baseline was $4.3( \pm 0.15)$ for the tamsulosin group, $4.9( \pm 0.15)$ for the dutasteride group, and $6.2( \pm 0.15)$ for the combination group ( $\mathrm{p}<0.001)$. A statistically significant difference was achieved for combination therapy over dutasteride alone from month 3 and over tamsulosin alone from month 9 . The percentage of men classified as IPSS responders at 24 month was significantly greater in the combination group for all three criteria (each comparison $\mathrm{p}<0.001)$. The increase in the peak urinary flow rate of the combination group was $2.4 \mathrm{~mL} / \mathrm{s}( \pm 0.12)$, significantly greater than $1.9 \mathrm{~mL} / \mathrm{s}( \pm 0.12)$ and $0.9 \mathrm{~mL} / \mathrm{s}$ $( \pm 0.12)$ in the dutasteride group and the tamsulosin group, respectively (each $\mathrm{p}<0.003$ ). The proportion of patients characterized as peak urinary flow rate responders was greatest in the combination group. Mean decrease from baseline in total prostate volume was $26.9 \%( \pm 0.62)$ in the combination therapy group, $28.0 \%( \pm 0.61)$ in the dutasteride group, and $0.0 \%( \pm 0.84)$ in the tamsulosin group. Drug related adverse events were significantly greater in the combination therapy group compared to either monotherapy group, but $\leq 5 \%$ of men in each group withdrew from the study due to these events. Prostate cancer was reported in 58 men (21 in the combination group, 11 in the dutasteride group, and 26 in tamsulosin group). No cases of IFIS were reported. ${ }^{21}$

\section{Conclusions}

Dutasteride is the only dual inhibitor of $5 \alpha$-reductase approved for use in the treatment of men with symptomatic BPH/BPE. Few drug interactions have been identified; however the use of this drug is contraindicated in men under age 18 and in women owing to potential adverse effects on normal development of the male reproductive tract. Side effects occur in less than 5\% and include impotence, loss of libido, ejaculatory dysfunction and gynecomastia. There is a potential risk of developing high-grade prostate cancer, although the overall risk of prostate cancer is reduced. Ongoing trials may support the use of dutasteride in the prevention of prostate cancer (as with finasteride in the PCPT) and its effects on prostate cancer require further evaluation. Treatment in men with moderate to severe lower urinary tract symptoms results in improved symptoms, increased urinary flow rate, a reduced risk of urinary retention and need for surgical intervention, and an improvement in quality of life.

Tamsulosin is a competitive antagonist of $\alpha_{1 \mathrm{~A}}$ adrenoreceptors in the prostate and bladder neck. It has been shown in numerous studies to significantly improve symptom scores and peak urinary flow rates in men with symptomatic BPH/ BPE and long-term studies suggest these effects are durable. Drug interactions are rare and serious side effects occur in less than $2 \%$ of patients in any treatment group. Patients treated with tamsulosin who undergo cataract surgery should advise their ophthalmologist because of the risk of IFIS.

Two-year data from the multicenter CombAT study shows that combination therapy with dutasteride and tamsulosin results in significantly greater decreases in IPSS and significant increases in peak urinary flow rates versus monotherapy with either dutasteride or tamsuolosin. Additionally, a significantly greater reduction in total prostate and transition zone volume was achieved in the combination group as compared to monotherapy with tamsulosin. Adverse drug events are more common with combination therapy but study withdraw rates are similar among the treatment groups and are less than $5 \%$.

\section{Disclosures}

The authors declare no conflicts of interest. 


\section{References}

1. Ball AJ, Fenely RCL, Abrams PH. The natural history of untreated "prostatism". BJU Int. 1981;53:613-616.

2. Jacobsen SJ, Girman CJ, Guess HA, et al. Natural history of prostatism: Longitudinal changes in voiding symptoms in community dwelling men. J Urol. 1996;155:595-600.

3. Arrighi HM, Metter EJ, Guess HA, et al. Natural history of benign prostatic hyperplasia and the risk of prostatectomy. The Baltimore Longitudinal Study of Aging. Urology. 1991;38(Suppl 1):4-8.

4. Roehrborn C, McConnell J, Barry M, et al. AUA guideline on management of benign prostatic hyperplasia (2003). Chapter 1: Diagnosis and treatment recommendations. J Urol. 2003;170: 530-547.

5. Roehrborn CG, Schwinn DA. $\alpha 1$-Adrenergic receptors and their inhibitors in lower urinary tract symptoms and benign prostatic hyperplasia. J Urol. 2004;171:1029-1035.

6. Lepor H. Phase III multicenter placebo-controlled study of tamsulaosin in benign prostatic hyperplasia. Tamsulosin Investigator Group. Urol. 1998;51:892-900.

7. Thompson IM, Goodman PJ, Tangen CM, et al. The influence of finasteride on the development of prostate cancer. $N$ Engl J Med. 2003;349:215-224.

8. Abromocicz, M, ed. Dutasteride (Avodart) for benign prostatic hyperplasia. Med Lett Drug Ther. 2002;44:109-110.

9. Bramson HN, Herman D, Batchelor KW, et al. Unique preclinical characteristics of GG745, a potent dual inhibitor of 5AR. J Pharmacol Exp Ther. 1997;282:1946-1502.

10. Roehrborn CG, Boyle P, Nickel JC, et al. Efficacy and safety of a dual inhibitor of 5-alpha-reductase types 1 and 2 (dutasteride) in men with benign prostatic hyperplasia. Urol. 2002;60:434-441.

11. AVODART (dutasteride): Prescribing information. In: Physicians' Desk Reference, 58th ed. Montvale, NJ: Thompson PDR; 2004. p. 1456-1459.
12. Roehrborn CG, Lukkarinen O, Mark S, et al. Long-term sustained improvement in symptoms of benign prostatic hyperplasia with the dual $5 \alpha$-reductase inhibitor dutasteride: results of 4-year studies. BJU Int. 2005;96:572-577.

13. Schulman C, Pommerville P, Hofner, K, et al. Long-term therapy with dual $5 \alpha$-reductase inhibitor dutasteride is well tolerated in men with symptomatic benign prostatic hyperplasia. BJU Int. 2006;97: 73-79.

14. O'Leary MP, Roehrborn C, Andriole G, et al. Improvements in benign prostatic hyperplasia-specific quality of life with dutasteride, the novel

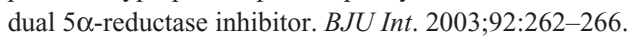

15. Desgrandchamps F, Droupy S, Irani J, et al. Effect of dutasteride on the symptoms of benign prostatic hyperplasia, and patient quality of life and discomfort in clinical practice. BJU Int. 2006;98:83-88.

16. Bostwick DG, Qian J, Civantos F, et al. Does finasteride alter the pathology of the prostate and cancer grading? Clin Prostate Cancer. 2004;2:228-235.

17. Gomella LG. Chemoprevention using dutasteride: The Reduce Trial. Curr Opin Urol. 2005;15:29-32.

18. Merrick GS, Butler WM, Wallner KE, et al. Efficacy of neoadjuvant bicalutamide and dutasteride as a cytoreductive regimen before prostate brachytherapy. Urol. 2006;68:116-120.

19. Narayan P, Tunuguntla HSGR. Long-term efficacy and safety of tamsulosin for benign prostatic hyperplasia. Rev Urol. 2005;7(Supp 4): S42-S48.

20. McConnell JD, Roehrborn CG, Bautista OM, et al. The long-term effect of doxazosinzocin, finasteride, and combination therapy on the clinical progression of benign prostatic hyperplasia. $N$ Engl J Med. 2003;349:2449-2451.

21. Roehrborn CG, Siami P, Barkin J, et al. The effects of dutasteride, tamsulosin and combination therapy on lower urinary tract symptoms in men with benign prostatic hyperplasia and prostatic enlargement: 2-year results from the CombAT study. J Urol. 2008;179:616-621.
Clinical Interventions in Aging

\section{Publish your work in this journal}

Clinical Interventions in Aging is an international, peer-reviewed journal focusing on evidence-based reports on the value or lack thereof of treatments intended to prevent or delay the onset of maladaptive correlates of aging in human beings. This journal is indexed on PubMed Central, MedLine, the American Chemical Society's 'Chemical

\section{Dovepress}

Abstracts Service' (CAS), Scopus and the Elsevier Bibliographic databases. The manuscript management system is completely online and includes a very quick and fair peer-review system, which is all easy to use. Visit http://www.dovepress.com/testimonials.php to read real quotes from published authors. 